\title{
Efficacy of Mass Quarantine as Leverage of Health System Governance During COVID-19 Outbreak: A Mini Policy Review
}

\author{
Mohammad Hossein Taghrir, MD ${ }^{1 *}$; Hossein Akbarialiabad, MD; Milad Ahmadi Marzaleh, PhD² \\ ${ }^{1}$ Student Research Committee, Shiraz University of Medical Sciences, Shiraz, Iran \\ ${ }^{2}$ Student Research Committee, Department of Health in Disasters and Emergencies, Health Human Resources Research Center, School of \\ Management and Medical Informatics, Shiraz University of Medical Sciences, Shiraz, Iran
}

\begin{abstract}
On January 23, 2020, the Chinese government announced the city lockdown of Wuhan. Since then, there have been controversial debates among experts about the efficacy of mass quarantine, the oldest and probably one of the most effective methods for controlling infectious disease outbreaks. The impact of health policymaking section of health system governance becomes visible to all stakeholders and the public in such emergency contexts. The success and failure of such policies should be evaluated in order to find the proper course of action for the local and international communities. In this review, we aim to investigate the efficacy of mass quarantine in China during the coronavirus disease 2019 (COVID-19) pandemic. We found good quality evidence for the effectiveness of mass quarantine during the current stage of COVID-19 pandemic, and these strategies seem to have been highly effective in controlling the spread of the disease.

Keywords: COVID-19, Disease outbreaks, Health Policy, Pandemics, Quarantine, Viruses

Cite this article as: Taghrir MH, Akbarialiabad H, Ahmadi Marzaleh M. Efficacy of mass quarantine as leverage of health system governance during COVID-19 outbreak: a mini policy review. Arch Iran Med. 2020;23(4):265-267. doi: 10.34172/aim.2020.08.
\end{abstract}

Received: March 20, 2020, Accepted: March 30, 2020, ePublished: April 1, 2020

\section{Introduction}

Quarantine has been a widely implicated method for many decades as one of the oldest and most effective methods for controlling communicable disease outbreaks. Quarantine is defined as the restriction of persons who have been exposed to a contagious disease, and Isolation is defined as the separation of ill individuals. However, these two words are being used interchangeably nowadays. ${ }^{1}$

A novel coronavirus named severe acute respiratory syndrome coronavirus 2 (SARS-Cov2) ${ }^{2}$ emerged as the cause of viral pneumonia in Wuhan, China, in December 2019. The World Health Organization (WHO) called it coronavirus disease 2019 or "COVID-19" and subsequently declared it a pandemic state on March 11, 2020..$^{3}$ As of March 27, 2020, more than 500000 cases of COVID-19, including 23000 deaths, have been reported from more than 180 countries and territories globally. China was the first government to impose quarantines and travel bans in Wuhan on January 23, 2020 and then throughout the country. They noticed that the disease would hardly be controlled without such strict restrictions. ${ }^{4}$ The efficacy of health policies has always been of importance, especially during contagious disease outbreaks, and it deserves investigation.

\section{Why Is This Review Needed?}

Quarantine is one of the most feared and misunderstood methods of controlling infectious disease epidemics, which may have significant psychological, emotional, and financial implications on quarantined families and patients. The psychological effects may include conditions such as acute stress disorder, post-traumatic stress disorder, major depression disorder, insomnia, irritability, and feeling of anger that have been discussed before. ${ }^{5}$ Considering the spread of COVID-19 throughout the majority of countries, health policymakers need compelling evidence of the previously implemented policies on this similar occasion. In the present study, we aim to investigate the efficacy of the mass quarantine policy applied in China during the COVID-19 outbreak.

\section{Efficacy of Mass Quarantine}

In order to evaluate the effectiveness of quarantine, some studies used Susceptible-Exposed-Infectious-Recovered (SEIR) model, which represents how fast people move from being susceptible to exposed, from exposed to infected, and from infected to recovered state. Using this model, Jia et al showed that the strict quarantine strategies in China such as home quarantine, traffic restrictions and travel bans, extension of the Chinese New Year vacations and delay in returning to work significantly decreased the transmission of infection in the community and were highly successful in controlling the initial stages of epidemic spreading. ${ }^{6}$ Another study performed by Li et al 
using the same model $^{7}$ revealed that quarantine strategies are probably more practical than traffic blockage control to reduce the spread of COVID-19; with more rigorous quarantine to the optimal rate of $100 \%$, it is predicted that the number of cases will decline by $89.7 \%$ A study by Li et al similarly showed that the epidemic size could probably decline to $87 \%$ by $100 \%$ quarantine effectiveness in the Hubei province, China. ${ }^{8}$ Although the optimal rate of quarantine application is not readily feasible, these analyses illustrate the probable efficiency of the recent mass quarantine.

In the study mentioned above, it was also confirmed that in the Hubei province, with one week delay in quarantine implementation, the number of cases would increase by $-10 \%$. On the contrary, it was estimated that with the earlier implementation of the quarantine by one and two weeks, the rate might decrease by $-25 \%$ and $-57.3 \%$, respectively. Using the modified-SEIR model, Yang et $\mathrm{al}^{9}$ showed that policies such as mass quarantine, strict travel limitation and large-scale monitoring of suspected cases were victorious in reducing the epidemic size and a delay in implementation of such policies by five days would have increased the epidemic size by three-folds in mainland China. Another study by Qiu and $\mathrm{Xiao}^{10}$ using the $\operatorname{SEIO}(\mathrm{MH})$ model showed that Wuhan lockdown decreased the R0 from 2.65 to 1.98 . It was predicted that by implementing city lockdown seven days earlier, the total number of infected people would have dropped by $72 \%$. Delaying 1-6 days would expand the epidemic size by even 5-times, while with 7 days of postponing lockdown implementation, the epidemic would be out of control.

Moreover, several studies used metapopulation models. Wu et $\mathrm{al}^{11}$ estimated that a $50 \%$ reduction in inter-city mobility would have a negligible effect. Also, with 25\% transmissibility reduction and 50\% reduction in intercity transmission, the peak of confirmed cases will dip by $50 \%$ in Wuhan city. Another study ${ }^{12}$ showed that the travel restriction on Wuhan has only delayed the epidemic progression in mainland China by 35 days without any noticeable effect on the epidemic size reduction but has had a more significant impact on the international scale, where case importations until mid-February were reduced by $-80 \%$.

A study by $\mathrm{Ai}$ et $\mathrm{al}^{13}$ used population outflow index and illustrated that the policy of city closure was successful by itself and earlier implementation would have been more productive. In the mentioned study, 687 and 1420 cases would have been diminished by implementing city closure one and two days in advance, respectively, and there would have been 722 and 1462 more cases if city closure had been postponed one and two days respectively. Chen et $\mathrm{al}^{14}$ showed that data from population migration and the number of confirmed cases showed that after lockdown of Wuhan and 19 cities of Hubei province, the outbreak burst was prevented and the number of cases increased only steadily, while it had been growing exponentially before those strategies.

On the opposite side, in a study by Read ${ }^{15}$ at the early stage of epidemic in China, it was estimated that with an ideal $99 \%$ travel restriction, the size of the outbreak outside of Wuhan might only have been reduced by $24.9 \%$. Thus, the effectiveness of travel restrictions is slight or unproven.

Another study by Zhang et $\mathrm{al}^{16}$ using the susceptibleexposed-infected-quarantined-recovered (SEIQR) model, found that social distancing (actions which increase physical distance between individuals such as canceling public activities, and postponing festivals and school reopening) could significantly mitigate the epidemic size in mainland China and epicenter lockdown would partially neutralize this favorable effect. Therefore, social distancing strategy without epicenter lockdown would be more feasible and cost-effective.

In the reviewed studies, mitigation methods other than quarantine have been suggested. A study conducted by Maier and Brockmann ${ }^{17}$ provided pieces of evidence that for breaking the transmission chain, mitigation strategies have to focus on the susceptible population and induce behavioral changes in them. Hellewell et $\mathrm{al}^{18}$ tried to investigate the effectiveness of case isolation and contact tracing using several scenarios with different initial cases and R0. They found that highly effective contact tracing and case isolation are sufficient to control the outbreak of COVID-19 within three months. But the amount of contacts needed to be isolated and traced differed between scenarios. For instance, a $90 \%$ probability of achieving control of an outbreak requires tracing and isolation of $80 \%$ of contacts for scenarios with a reproduction number of 2.5 .

\section{Discussion and Conclusion}

Based on the above, the majority of studies confirmed the positive effect of implementing mass quarantine and movement limitations in China during the COVID-19 epidemic. However, two studies showed that travel restriction and city lockdown were not highly efficient. Meanwhile, two studies also suggested effective methods other than quarantine without mentioning the success or failure of quarantine.

Compared to other emerging infectious diseases, for instance, severe acute respiratory syndrome (SARS) in 2003, Canada, China and Taiwan were the countries hardest hit by SARS. About 30000 persons were quarantined in Toronto, Canada where the number of probable cases were 250 , in contrast to Beijing, China which had a similar number of quarantined persons but with 2500 probable cases. ${ }^{19}$ Richard Schabas noted that mass quarantine during the SARS outbreak in Toronto was unnecessary on a large scale, ineffective in finding suspected cases and played little role in controlling the outbreak. ${ }^{20}$ One study investigated the efficiency of quarantine during the SARS epidemic in 
Beijing, China in $2003 .{ }^{21}$ The results showed that mass quarantine was not needed and if the authorities focused on persons who had contact with an active SARS patient, quarantine could have been reduced by two thirds without compromised efficacy. In Taiwan, 131000 persons were quarantined during the SARS outbreak but only 12 were reported as possible SARS cases, and only two confirmed cases were found among those quarantined. It is believed that excessive use of quarantine has led to public fear and has thus proven counterproductive. ${ }^{22}$

The findings of the present review show good quality evidence for effectiveness of mass quarantine during the current stage of COVID-19 pandemic, unlike the experience of mass quarantine, as a wide community containment strategy during the SARS outbreak in 2003 which was reported to be ineffective. However, further investigations are required in the future. The present study aimed to review the effectiveness of mass quarantine during the COVID-19 epidemic in China. Implementation of these strategies in other countries during the current pandemic needs to consider other principles such as ethics and socioeconomic factors.

\section{Authors' Contribution}

MHT conceived and developed the presented idea and wrote the primary draft. HA and MAM helped in completing the draft. All authors provided critical feedback, discussed the results and contributed to the final manuscript.

\section{Conflict of Interest Disclosures}

We know of no conflicts of interest associated with this publication and there has been no significant financial support for this work that could have influenced its outcome.

\section{Ethical Statement}

Not applicable.

\section{Acknowledgements}

Special thanks to Dr. Ramin Shiraly MD (Associate Professor of Community Medicine, Department of Community Medicine, School of Medicine, Shiraz University of Medical Sciences) for supervising the project and editing the final version of manuscript.

\section{References}

1. Cetron M, Landwirth J. Public health and ethical considerations in planning for quarantine. Yale J Biol Med. 2005;78(5):329-34.

2. Gorbalenya AE. Severe acute respiratory syndrome-related coronavirus-The species and its viruses, a statement of the Coronavirus Study Group. BioRxiv. 2020. doi: 10.1101/2020.02.07.937862.

3. WHO. WHO characterizes COVID-19 as a pandemic [updated March 11, 2020. Available from: https://www.who.int/ emergencies/diseases/novel-coronavirus-2019/events-as-theyhappen.

4. Wu J, Gamber M, Sun W. Does Wuhan Need to be in Lockdown during the Chinese Lunar New Year? Int J Environ Res Public Health. 2020;17(3). doi: 10.3390/ijerph17031002.

5. Brooks SK, Webster RK, Smith LE, Woodland L, Wessely S, Greenberg N, et al. The psychological impact of quarantine and how to reduce it: rapid review of the evidence. Lancet. 2020. doi: 10.1016/S0140-6736(20)30460-8.

6. Jia J, Ding J, Liu S, Liao G, Li J, Duan B, Wang G, Zhang R. Modeling the control of COVID-19: impact of policy interventions and meteorological factors. Electron J Differential Equations. 2020;2020(23):1-24.

7. Li D, Liu Z, Liu Q, Gao Z, Zhu J, Yang J, et al. Estimating the Efficacy of Traffic Blockage and Quarantine for the Epidemic Caused by 2019-nCoV (COVID-19). medRxiv. 2020. doi: 10.1101/2020.02.14.20022913.

8. Li R, Lu W, Yang X, Feng P, Muqimova O, Chen X, et al. Prediction of the Epidemic of COVID-19 Based on Quarantined Surveillance inChina.medRxiv.2020.doi:10.1101/2020.02.27.20027169.

9. Yang Z, Zeng Z, Wang K. Modified SEIR and AI prediction of the epidemics trend of COVID-19 in China under public health interventions. J Thorac Dis. 2020. doi: 10.21037/jtd.2020.02.64

10. Qiu T, Xiao H. Revealing the Influence of National Public Health Response for the Outbreak of the SARS-CoV-2 Epidemic in Wuhan, China Through Status Dynamic Modeling. 2020. doi: $10.2139 / \mathrm{ssrn} .3550027$.

11. $\mathrm{Wu} J \mathrm{~T}$, Leung $\mathrm{K}$, Leung GM. Nowcasting and forecasting the potential domestic and international spread of the 2019nCoV outbreak originating in Wuhan, China: a modelling study. Lancet. 2020;395(10225):689-97. doi: 10.1016/S01406736(20)30260-9.

12. Chinazzi M, Davis JT, Ajelli M, Gioannini C, Litvinova M, Merler S, et al. The effect of travel restrictions on the spread of the 2019 novel coronavirus (COVID-19) outbreak. Science. 2020. doi: 10.1126/science.aba9757.

13. Ai S, Zhu G, Tian F, Li H, GaoY, WuY, et al. Population movement, city closure and spatial transmission of the 2019-nCoV infection inChina. medRxiv.2020. doi:10.1101/2020.02.04.20020339

14. Chen Z, Zhang Q, Lu Y, Zhang X, Zhang W, Guo C, et al. Distribution of the 2019-nCoV Epidemic and Correlation with Population Emigration from Wuhan, China. medRxiv. 2020. doi: $\quad 10.1101 / 2020.02 .10 .20021824$.

15. Read JM, Bridgen JR, Cummings DA, Ho A, Jewell CP. Novel coronavirus 2019-nCoV: early estimation of epidemiological parameters and epidemic predictions. medRxiv. 2020. doi: 10.1101/2020.01.23.20018549.

16. Zhang Y, Jiang B, Yuan J, Tao Y. The impact of social distancing and epicenter lockdown on the COVID-19 epidemic in mainland China: A data-driven SEIQR model study. medRxiv. 2020. doi: 10.1101/2020.03.04.20031187.

17. Maier BF, Brockmann D. Effective containment explains sub-exponential growth in confirmed cases of recent COVID-19 outbreak in Mainland China. medRxiv. 2020. doi: 10.1101/2020.02.18.20024414

18. Hellewell J, Abbott S, Gimma A, Bosse NI, Jarvis Cl, Russell TW, et al. Feasibility of controlling COVID-19 outbreaks by isolation of cases and contacts. Lancet Glob Health. 2020. doi: 10.1016/ S2214-109X(20)30074-7.

19. Naylor CD. Learning from SARS: renewal of public health in Canada: a report of the National Advisory Committee on SARS and Public Health: National Advisory Committee; 2003. Available from: https://www.canada.ca/content/dam/phac-aspc/ migration/phac-aspc/publicat/sars-sras/pdf/sars-e.pdf.

20. Schabas R. Severe acute respiratory syndrome: Did quarantine help? Can J Infect Dis Med Microbiol. 2004;15(4):204. doi: 10.1155/2004/521892.

21. Ou J, Li Q, Zeng G, Dun Z. Efficiency of quarantine during an epidemic of severe acute respiratory syndrome-Beijing, China, 2003. MMWR Morb Mortal Wkly Rep. 2003;52(43):1037-1040.

22. Control CfD, Rothstein MA, Alcalde MG, Elster NR, Majumder MA, Palmer LI, et al. Quarantine and isolation: Lessons learned from SARS: University of Louisville School of Medicine, Institute for Bioethics, Health; 2003. Available from: https://stacks.cdc. gov/view/cdc/11429/cdc_11429_DS1.pdf. 